\title{
Ectopic phytocystatin expression increases nodule numbers and influences the responses of soybean (Glycine max) to nitrogen deficiency
}

\author{
Marian D. Quain ${ }^{\text {a,b,1 }}$, Matome E. Makgopa ${ }^{\text {a,c,1 }}$, James W. Cooper ${ }^{\text {a }}$, Karl J. Kunert ${ }^{\text {a,c }}$, Christine H. Foyer ${ }^{\text {a,* }}$ \\ a Centre for Plant Sciences, Faculty of Biology, University of Leeds, Leeds LS2 9JT, UK \\ b Council for Scientific and Industrial Research, Crops Research Institute, P.O. Box 3785, Kumasi, Ghana \\ C Forestry and Agricultural Biotechnology Institute, Plant Science Department, University of Pretoria, Pretoria 0002, South Africa
}

Keywords:

Cystatin

Cysteine protease

Lateral root density

Leaf senescence

Nitrogen deficiency

Nodulation

Vacuolar processing enzymes

\begin{abstract}
A B S T R A C T
Cysteine proteases and cystatins have many functions that remain poorly characterised, particularly in crop plants. We therefore investigated the responses of these proteins to nitrogen deficiency in wild-type soy-beans and in two independent transgenic soybean lines (OCI-1 and OCI-2) that express the rice cystatin, oryzacystatin-I (OCI). Plants were grown for four weeks under either a high (5 mM) nitrate (HN) regime or in the absence of added nitrate (LN) in the absence or presence of symbiotic rhizobial bacteria. Under the LN regime all lines showed similar classic symptoms of nitrogen deficiency including lower shoot bio-mass and leaf chlorophyll. However, the $\mathrm{LN}$-induced decreases in leaf protein and increases in root protein tended to be smaller in the $\mathrm{OCI}-1$ and $\mathrm{OCI}-2$ lines than in the wild type. When LN-plants were grown with rhizobia, OCI-1 and OCI-2 roots had significantly more crown nodules than wild-type plants. The growth nitrogen regime had a significant effect on the abundance of transcripts encoding vacuolar processing enzymes (VPEs), LN-dependent increases in VPE2 and VPE3 transcripts in all lines. However, the LN-depen-dent increases of VPE2 and VPE3 transcripts were significantly lower in the leaves of OCI-1 and $\mathrm{OCI}-2$ plants than in the wild type. These results show that nitrogen availability regulates the leaf and root cysteine pro-tease, VPE and cystatin transcript profiles in a manner that is in some cases influenced by ectopic OCI expression. Moreover, the OCI-dependent inhibition of papain-like cysteine proteases favours increased nodulation and enhanced tolerance to nitrogen limitation, as shown by the smaller LNdependent decreases in leaf protein observed in the OCI- 1 and OCI- 2 plants relative to the wild type.
\end{abstract}

\section{Introduction}

The global population continues to rise by about eighty million people per year (Park et al., 2011). There is therefore an urgent need for improved varieties of crops, such as soybean that can sus-tain high yields even on poor soils (Tilman et al., 2011; Park et al., 2011). The production of improved crops with improved nitrogen use efficiencies so that they are better able to withstand low soil

Abbreviations: CP, cysteine protease; CYS, cystatin; OCI, oryzacystatin-I; VPE, vacuolar processing enzyme.

* Corresponding author. Tel.: +44 113343 1421; fax: +44 01133432882.

E-mail address: c.foyer@leeds.ac.uk (C.H. Foyer)

All the work was conducted in the University of Leeds but the authors have now

returned to their home institutions as identified in affiliations b and $c$. nitrogen availability is an essential requirement for future agricul-ture worldwide (Kant et al., 2010).

Grain and forage legumes account for nearly thirty percent of the worlds' primary crop production and are important contributors to the dietary protein intake of humans and animals (Graham and Vance, 2003). For example, soybean seeds provide more than $35 \%$ of the world's processed vegetable oil, which is used in bread and margarine production as well as industrial products (Van Heerden et al., 2003). Despite the high economic value of soybean, increases in yields have fallen behind those of other staple crops, such as cereals (Jeuffroy and Ney, 1997). This is particularly true in developing countries, where unfavourable environmental conditions can limit legumes productivity (Graham and Vance, 2000). Like other legumes, soybean is able 
to form a mutually-beneficial symbiotic relationship with nitrogenfixing bacteria (Oldroyd et al., 2011). Symbiotic nitrogen fixation occurring in soybean root nodules that house the bacteria supports high crop productivity even in nitrogen-poor soils (Moulin et al., 2001).

Nitrogen has long been recognised as one of the most important macronutrients required to sustain plant growth and a paradigm of plant responses to nitrogen limitation has been established that includes decreased photosynthesis, increased tis-sue carbon/ nitrogen ratios, decreased shoot/root ratios and leaf carbohydrate accumulation, as well as extensive reprogramming of gene expression (Scheible et al., 2004; Bi et al., 2007). The remobilisation of nitrogen stored in photosynthetic proteins is of particular importance in supporting plant growth and develop-ment in situations of nitrogen limitation. Hence large increases in the abundance of transcripts encoding proteins involved in protein turnover and leaf senescence are characteristic of the nitrogen deficiency response (Crafts-Bradner et al., 1998; Horstensteiner and Feller, 2002).

Proteolysis, catalysed by the proteasome and a raft of different proteases, underpins leaf nitrogen remobilisation (Grudkowska and Zagdanska, 2004). Proteases fulfil many important functions in plants including the maturation and removal of abnormal or damaged proteins (Forsthoefel et al., 1998; Solomon et al., 1999; Grudkowska and Zagdanska, 2004). They are also crucial for the restructuring of the composition of the cellular protein network in response to developmental triggers and environmental stimuli. Cysteine proteases are involved in protein remobilisation and nitrogen recycling occurring during leaf senescence and programmed cell death for seed development (Beers et al., 2000; Kato et al., 2003; Esteban-Garcia et al., 2010). For example, the vac-uolar processing enzyme (VPE) family of cysteine proteases, which are also known as legumains, play a crucial role in organ senes-cence and cell death. Loss of VPE function prevents cell death dur-ing the hypersensitive response to pathogens (Hara-Nishimura et al., 2005; Hatsugai et al., 2006). VPEs resemble mammalian caspases and they are localised in the vacuole. Their functions have been wellcharacterized in maturing seeds, where they play a key role in the processing of storage proteins (Shimada et al., 2003). They are able to activate pre-proteases by post-translational mod-ification (Roberts et al., 2012).

The activity of cysteine proteases is regulated in situ by interactions with tight-binding inhibitors called cystatins. Cystatins inhibit cysteine proteases of the papain C1A family in a revers-ible manner (Martinez and Diaz, 2008; Martinez et al., 2012). The rice endosperm cystatin, called oryzacystatin I (OCI), com-prises 102 amino acids and has a tertiary structure consisting of a central $\alpha$ helix and a five-stranded anti-parallel $\beta$-sheets with no disulphide bonds (Benchabane et al., 2010). OCI, which is perhaps the bestcharacterised plant cystatin to date, has a highly conserved QXVXG motif that is required for cysteine pro-tease inhibition (Arai et al., 1991; Jenko et al., 2003). Phytocyst-atins regulate cysteine protease-mediated protein turnover during growth and development. The coordinated expression of transcripts encoding cysteine protease and cystatin interacting partners has been reported in senescent spinach leaves (Tajima et al., 2011). Ectopic OCI expression not only altered the growth and development of tobacco (Van der Vyver et al., 2003; Prins et al., 2008), soybean and Arabidopsis thaliana plants (Quain et al., 2014) but also enhanced tolerance to abiotic stresses, such as low temperatures and drought (Prins et al., 2008; Quain et al.,

2014).

Soybeans can suffer from nitrogen deficiency under field conditions, particularly at flowering when the nodules start to senesce or when seeds are either planted without inoculation of soils with appropriate symbiotic bacteria, particularly in areas where soy- bean has not been grown previously, or on acid soils that prevent successful nodulation (Mengel and Ruiz-Diaz, 2012). Abiotic stresses, such as defoliation, drought and exposure to heavy metals, can cause premature nodule senescence resulting in impaired symbiotic nitrogen fixation (Gordon et al., 1990; Karina et al., 2003).

The recent release of the complete soybean genome (Schmutz et al., 2010) and the RNAseq atlas of genes expressed in fourteen different soybean tissues (Severin et al., 2010) allows the accurate identification and characterisation of soybean cystatins, cysteine proteases and VPEs. The Phytozome database (www.phytozome. net) currently contains over 300 cystatin-like sequences from the Viridiplantae kingdom, 706 C1 cysteine protease sequences as well as 362 C13 cysteine protease (VPE-type) sequences. However, the exact functions of most of these proteins remain largely uncharacterised (Severin et al., 2010). In this study, we selected a small number of model cysteine proteases, VPEs and cystatins that were reported to be expressed in leaves and roots in the Phytozome database. Our aim was to explore whether the levels of transcripts encoding these proteins was influenced by ectopic OCI expression in soybean plants that had been grown for four weeks with either a high (5 mM) nitrate (HN) supply or in the absence of added nitrate (LN). We selected two independent transgenic OCI expressing soybean lines (OCI-1 and OCI-2) that had different levels of OCI transcripts in the leaves (Quain et al., 2014). The variation in the levels of expression between different independent transgenic lines is consistent with the known features of 35S-driven transgene expression in $A$. thaliana, in which a bimodal expression pattern has been reported consisting of $20 \%$ high-level expressers and $80 \%$ lowlevel expressers (De Bolle et al., 2003). We compared the effects of ectopic OCI expression in the responses of soybeans to LN using these transgenic lines (Quain et al., 2014). Our earlier studies had indicated that OCI-1 line had about half the levels of the OCI protein in their leaves than the OCI-2 line (Quain et al., 2014). The data presented here show that growth under LN condi-tions induced changes in the levels of transcripts encoding cysteine proteases, VPEs and cystatins, and that the LN-dependent responses of the soybean plants are modified in the OCI-1 and OCI-2 lines.

\section{Experimental}

\subsection{Plant growth}

Soybean (Glycine max cultivar Williams) transformation was performed as described previously (Quain et al., 2014). Two independent transformed soybean lines (OCI-1 and $\mathrm{OCI}-2$ ) that had been produced by selfing the primary transformants twice were used in this study. The lines were selected on the basis of high OCI transcript abundance and OCI protein in leaves and other tis-sues (Quain et al., 2014). Seeds of wild-type soybeans and OCI-1 and OCI-2 lines were sown in pots containing vermiculite. A min-imum of twenty plants per line were analysed in each experiment. Plants were grown in a controlled environment chamber at day/night temperatures of $28{ }^{\circ} \mathrm{C} / 20^{\circ} \mathrm{C}$, respectively, and an irradiance of 400 $\mathrm{mol}^{-2} \mathrm{~s}^{-1}$ with a $12 \mathrm{~h}$ day/12 h night cycle. Plants were sup-plied daily with distilled water. $100 \mathrm{ml}$ full-strength Hoagland's solution per plant was added twice per week to plants grown under HN (high nitrogen) conditions while those grown under LN (low nitrogen) conditions were provided twice per week with nitrogenfree Hoagland's solution. Hoagland's solution consisted of $\mathrm{KNO}_{3}$ (5.0 mM), $\mathrm{MgSO}_{4} \cdot 7 \mathrm{H}_{2} \mathrm{O}(2 \mathrm{mM}), \mathrm{KH}_{2} \mathrm{PO}_{4}(1 \mathrm{mM})$, FeDTA $(0.1 \mathrm{mM})$, $\mathrm{CaCl}_{2} \cdot 2 \mathrm{H}_{2} \mathrm{O}(5 \mathrm{mM})$ and $\mathrm{KCl}(0.05 \mathrm{mM})$. Macronutrient salts were added to the solution $\left[\mathrm{H}_{3} \mathrm{BO}_{3}(46 \mu \mathrm{M}), \mathrm{MnSO}_{4} \cdot 4 \mathrm{H}_{2} \mathrm{O}(3.9 \mu \mathrm{M})\right.$, $\mathrm{ZnSO}_{4} \cdot 7 \mathrm{H}_{2} \mathrm{O}(3.9 \mu \mathrm{M}), \mathrm{CuSO}_{4} \cdot 5 \mathrm{H}_{2} \mathrm{O}(1 \mu \mathrm{M}), \mathrm{Na}_{2} \mathrm{MoO}_{4} \cdot 2 \mathrm{H}_{2} \mathrm{O}(0.1$ $\mu \mathrm{M})]$, deionised water was added to attain the required volume and the $\mathrm{pH}$ was adjusted to 6.8 with $1 \mathrm{M} \mathrm{NaOH}$. The $\mathrm{LN}$ 
Hoagland's solution had the same composition except that no $\mathrm{KNO}_{3}$ was added.

\subsection{Nodulation experiments}

For the nodulation experiments, soybean plants grown in the presence of Bradyrhizobia (Soygro Ltd., South Africa). Seeds were inoculated with the bacteria by adding the inoculant during germination under LN growth conditions. Nodule characteristics were determined from 6 individual plants grown for 8 weeks after inoculation with Bradyrhizobia.

\subsection{Phenotypic analysis}

Phenotypic analysis was performed on plants grown for 4 weeks as follows: shoot length and leaf number were recorded before leaf samples were collected for further analysis, as described below. Nodule numbers and diameter were determined on crown nodules. The diameter of crown nodules was measured around the widest point using a digital vernier calliper. The length of the main root and the number of first order lateral roots was recorded. Root densities were then calculated. The shoot and root systems of other sets of plants were weighed to obtain fresh weight values and then dried at $75{ }^{\circ} \mathrm{C}$ for two days before the determination of dry weights.

\subsection{Papain-like cysteine proteases activities}

The activity of papain-like cysteine proteases was measured on extracts made by grinding fresh shoots and roots in $50 \mathrm{mM}$ sodium phosphate buffer ( $\mathrm{pH}$ 6.0). Each experiment involved extracts from three plants per line. Assays were performed essentially as described by Salvesen and Nagase (1989) using a fluorogenic cathepsin L substrate (Z-Phe-Arg-MCA), which had been prepared in DMSO and stored as a $400 \mu \mathrm{M}$ stock solution. Root or shoot extracts $(1-10 \mu \mathrm{g} / \mu \mathrm{l}$ protein) were added to the assay media, which consisted of $50 \mathrm{mM}$ sodium phosphate buffer ( $\mathrm{pH}$ 6.0) con-taining 5 $\mathrm{mM}$ L-cysteine. The reactions were performed at $25{ }^{\circ} \mathrm{C}$ and started by the addition of $10 \mu \mathrm{M}$ Z-Phe-Arg-MCA. Protease activity was measured over a 20 min period. Fluorescence mea-surements were performed using a spectro-fluorometer (BMG Flu-oStar Galaxy, USA) with excitation and emission wavelengths at $340 \pm 10 \mathrm{~nm}$ and $450 \pm 10 \mathrm{~nm}$, respectively. The cysteine protease inhibitor E64 was added at a concentration of $10 \mathrm{nM}$ to inhibit activity and confirm cysteine protease-mediated catalysis. Reac-tion rates expressed as fluorescence units (FU/min) were deter-mined from the slopes of the reaction curves. Each sample was measured in triplicate.

\subsection{RNA isolation and $q P C R$}

Real-time (qPCR) was performed essentially as described previously by Pellny et al. (2009). RNA was extracted from the three plants representing each line (OCI-1, OCI-2 and wild-type control) previously used for cysteine protease activity measurement. RNA was extracted with the NucleoSpin RNA Plant kit (Thermo Scientific Abgene, UK) according to the protocol provided by the manufacturer. RNA reverse transcription and quantitative PCR was performed on an Eppendorf Realplex ${ }^{2}$ real-time PCR system by onestep RT-PCR with the Quantifast SYBR Green RT-PCR Kit (Quiagen, Germany) following manufacturer's instructions. Relative quantification and normalisation was done with the $\Delta \Delta \mathrm{Cq}$ method. Specificity of PCR amplification was confirmed by melting curve analysis $\left(75-95^{\circ} \mathrm{C}\right)$. Amplicon specificity was screened by BLAST searches to detect any off-targets. Reverse transcriptase samples minus controls were used once for each RNA sample to detect any genomic DNA contamination. All reactions for individ-ual RNA samples were setup in triplicates. The expression of the genes of interest were normalised with an endogenous control, the soybean elongation factor (ELFForward: GTTGAAAAGCC ACGGGACA; Reverse: TCTTACCCCTTGAGCGTGG). Accession num-bers and sequences of forward and reverse primers applied for papain-like cysteine proteases (CP), vacuolar processing enzymes (VPEs) and cystains (CYS) are provided in Table 1.

\subsection{Chlorophyll and protein measurements}

Leaf chlorophyll and protein contents were determined in leaf samples of plants used for analysis of plant characteristics that had been ground in liquid nitrogen. Pigments extracted in $96 \%$ (v/v) ethanol were determined according to Lichtenthaler (1987). The soluble protein content was determined according to the method of Bradford (1976) with BSA applied as a protein standard.

\subsection{Statistical analysis}

Data were analysed by ANOVA and the significance level was set at $5 \%$.

\section{Results}

\subsection{Confirmation of OCI transgene integration and expression}

PCR amplification of genomic DNA isolated from the cotyledons of wild-type soybean seedlings and the OCI-1 and OCI-2 lines was performed using the $\mathrm{OCI}$ primers shown in Table 1 . The expected $300 \mathrm{bp}$ fragment was amplified in the transgenic lines. Sequence analysis confirmed the OCI coding sequence in all of the plants from the $\mathrm{OCI}-1$ and $\mathrm{OCI}-2$ lines used in the following experiments.

Table 1

Primer sequences for the amplification of different VPEs, papain-like cysteine proteases, cystatins and $40 \mathrm{~S}$.

\begin{tabular}{|c|c|c|}
\hline Phytozome ID & Forward primer $\left(5^{\prime}-3^{\prime}\right)$ & Reverse primer $\left(3^{\prime}-5^{\prime}\right)$ \\
\hline $\mathrm{OCI}$ & ATG TCG AGC GAC GGA GGG & TTGCACTGGCTACGACAGGC \\
\hline Glyma05g04230 (VPE2) & CACCATCCCTTGTAAATTGT & GGGGTTTCAGTGCATAATAA \\
\hline Glyma14g10620 (VPE3) & GGTCGTGGATGTTGCTGAGG & ATCTGCTTGATGCCTGTAGTTTCC \\
\hline Glyma17g05670 (CP2) & GCTTGTCACTGCTCATTTTCGC & TTTTCCGGTGTAGGGATATGC \\
\hline Glyma10g35100 (CP3) & GAGGCCATGCCCTCATGT & TCACСTCTCTCCССAGTGTAGG \\
\hline Glyma14g40670 (CP4) & ATATGGAGCGTGTGACTCGG & GTAATATCCATTCTCTCCССАGСTC \\
\hline Glyma04g03090 (CP5) & AAGCTGTGGTGCATGTTGGG & AGTGGCGCTTGTCTTTGCAG \\
\hline Glyma13g04250 (CYS8) & ACTGCCGCGTGTAAAAACTT & TGAAATCGTGATTTGGCATC \\
\hline Glyma14g04260 (CYS9) & AAATGGTCGCAAGGTGAAAG & AGGATGCCCATTTGTACAGC \\
\hline Glyma18g00690 (CYS10) & CAGCAACAAGTGGTGTCAGG & CTACTGGATGCATGGTGGTG \\
\hline $40 \mathrm{~S}$ & GCCAGCCTGCTAACACTAAG & AAGAGTCTGAGTACGCACAAG \\
\hline
\end{tabular}



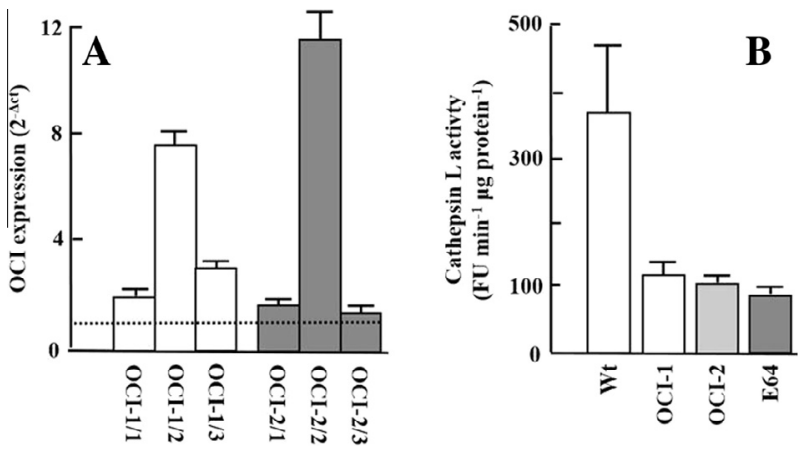

Fig. 1. (A) Relative abundance of OC-I transcripts in the leaves of three individual plants of the OCI- 1 (OCI-1/1, OCI- $1 / 2$, OCI- $1 / 3)$ and OCI-2 (OCI-2/1, OCI-2/2, OCI-2/ 3 ) lines measured under HN conditions. The dotted line indicates the level of transcripts encoding OCI-related endogenous soybean cystatins, which were also amplified by the primer pair applied for OCI amplification. This basal value was set at 1 . Bars are means \pm SD of three experiments. (B) Cathepsin L-like cysteine protease activities measured in cotyledons. Error bars indicate mean \pm SD of three biological replicates derived from leaf material measured in triplicate.

At the beginning of each experiment, qPCR analysis was performed on cotyledons to confirm the presence of OCI transcripts in the OCI-1 and OCI-2 plants, as illustrated in Fig. 1A. OCI transcripts were absent from the wild-type control plants. However, the OCI primers used in these studies were found not to be highly specific for OCI because some closely related endogenous soybean cystatins were also amplified in these studies. The abundance of these soybean OCI-related transcripts was therefore set at 1 according to values obtained in the wild type (Fig. 1A, dotted line). The relative abundance of OC-I transcripts measured in the OCI-1 and OCI-2 lines varied between plants and with the age of plants at sampling, as illustrated in Fig. 1A. Nevertheless, all the transgenic plants tested always expressed the $\mathrm{OCI}$ transgene, and the relative abundance of transcripts in the OCI-1 and OCI-2 tissues was similar at the point of measurement.

Cysteine protease activities were decreased in the cotyledons of the OCI- 1 and OCI- 2 plants (Fig. 1B) and plants of the OCI-1 and OCI-2 lines had significantly $(P \leqslant 0.05)$ lower cathepsin L cysteine protease activities (measured by the hydrolysis of the cathepsin-L substrate Z-Phe-Arg-MCA) than the wild-type plants (Fig. 1B). The level of OCI-dependent inhibition of cathepsin L cysteine pro-tease activities was similar that of the inhibition observed in wild-type plants resulting from the addition of the cysteine protease inhibitor E64 (Fig. 1B).

\subsection{Effects of ectopic $\mathrm{OCI}$ expression on crown nodules}

When OCI-1, OCI-2 and wild-plants were grown in the absence of added nitrogen, but in the presence of rhizobial bacteria, crown nodules were produced in all lines, as illustrated in Fig. 2A. While the crown nodules of the two OCI lines were significantly smaller $(P \leqslant 0.05)$ than those of the wild-type control (Fig. 2B), roots of the plants of OCI- 1 and $\mathrm{OCI}-2$ lines had significantly $(P \leqslant 0.05)$ more crown nodules than wild-type plants (Fig. 2C).

\subsection{Low nitrogen-dependent changes in root architecture}

The leaves of the plants grown for four weeks in the absence of nitrogen were visibly less green than the leaves of plants grown with high ( $5 \mathrm{mM}$ ) nitrate (Fig. 3A) and they had also lower leaf chlorophyll contents (Fig. 3B). The plants grown for four weeks in the absence of nitrogen had further fewer leaves than those grown under HN (Fig. 3C). Shoot height was similar in all lines
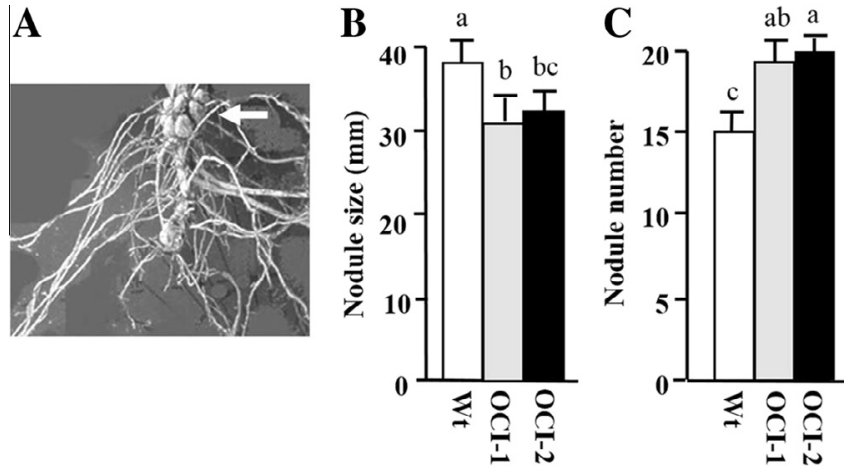

Fig. 2. Root crown nodules of soybean indicated by arrow (A) and crown nodule size and nodule size (B) and number (C) of wild-type soybean plants and plants of transgenic lines OC-I and OC-2 grown under low nitrogen for 8 weeks. Values represent the mean of 92 crown nodules from wild-type, 119 nodules from OCI-1 and 120 nodules from OCI-2 plants harvested from 6 individual plants \pm SD. Different letters denote significant differences between the different types of plants at $P \leqslant 0.05$

under both growth conditions (Fig. 3D). The shoots of the OCI-1 and OCI-2 lines had significantly higher dry matter accumulation than the wild type under $\mathrm{HN}$ conditions (Fig. 3E). While the shoot biomass was decreased to a similar extent in all lines as a result of growth under LN (Fig. 3E), the LN-dependent decrease in leaf protein was less marked in the OCI-1 and $\mathrm{OCI}-2$ lines than in the wild-type line (Fig. 3F).

Root biomass was significantly increased $(P \leqslant 0.05)$ under $\mathrm{LN}$ compared to HN conditions (Fig. 4A). However, root biomass was similar in all lines under both the HN and LN growth regimes (Fig. 4A). Root protein levels expressed on a fresh weight basis were significantly increased $(P \leqslant 0.05)$ under $\mathrm{LN}$ compared to HN conditions, and there were no consistent differences between the protein contents of OCI- 1 and OC-2 lines and the wild-type roots (Fig. 4B). While lateral root density was significantly $(P \leqslant 0.05)$ increased under LN compared to HN conditions in all the soybean lines, there were no consistent differences in the LN-induced increase in lateral root density in the OCI-1 and OC-2 lines compared to the wild-type roots (Fig. 4C).

3.4. Low nitrogen-dependent changes in papain-like cysteine protease, VPE and cystatin transcripts

Growth under LN conditions altered the abundance of transcripts encoding papain-like cysteine proteases VPEs and cystatins (Table 2). Table 2 compares the expression of genes that had previously been measured in young leaves and roots by RNAseq analysis (Severin et al., 2010). The data presented here using qRTPCR analysis provide evidence of changes in transcripts that were below the levels of detection by the RNAseq technique in non-treated tissue samples (Severin et al., 2010). Transcripts encoding the papain-like cysteine proteases CP2, CP3, CP4 and CP5 were either unchanged, or decreased, in abundance under LN when compared to HN conditions in leaves and roots with the exception of root CP4 of line OCI-1 where transcription increased under LN (Fig. 5). The abundance of CP2 and CP5 transcripts was lower in leaves (Fig. 5A and D) and roots (Fig. 5E and H) under LN compared to HN conditions. The abundance of CP3 transcripts (that have not previously not been detected in leaves e.g., by Severin et al., 2010; Table 2) was low under HN conditions in leaves. CP3 transcripts were below the level of detection under LN conditions (Fig. 5B). Low levels of CP3 transcripts were determined in roots by RNAseq (Table 2). The abundance of CP3 transcripts in roots 

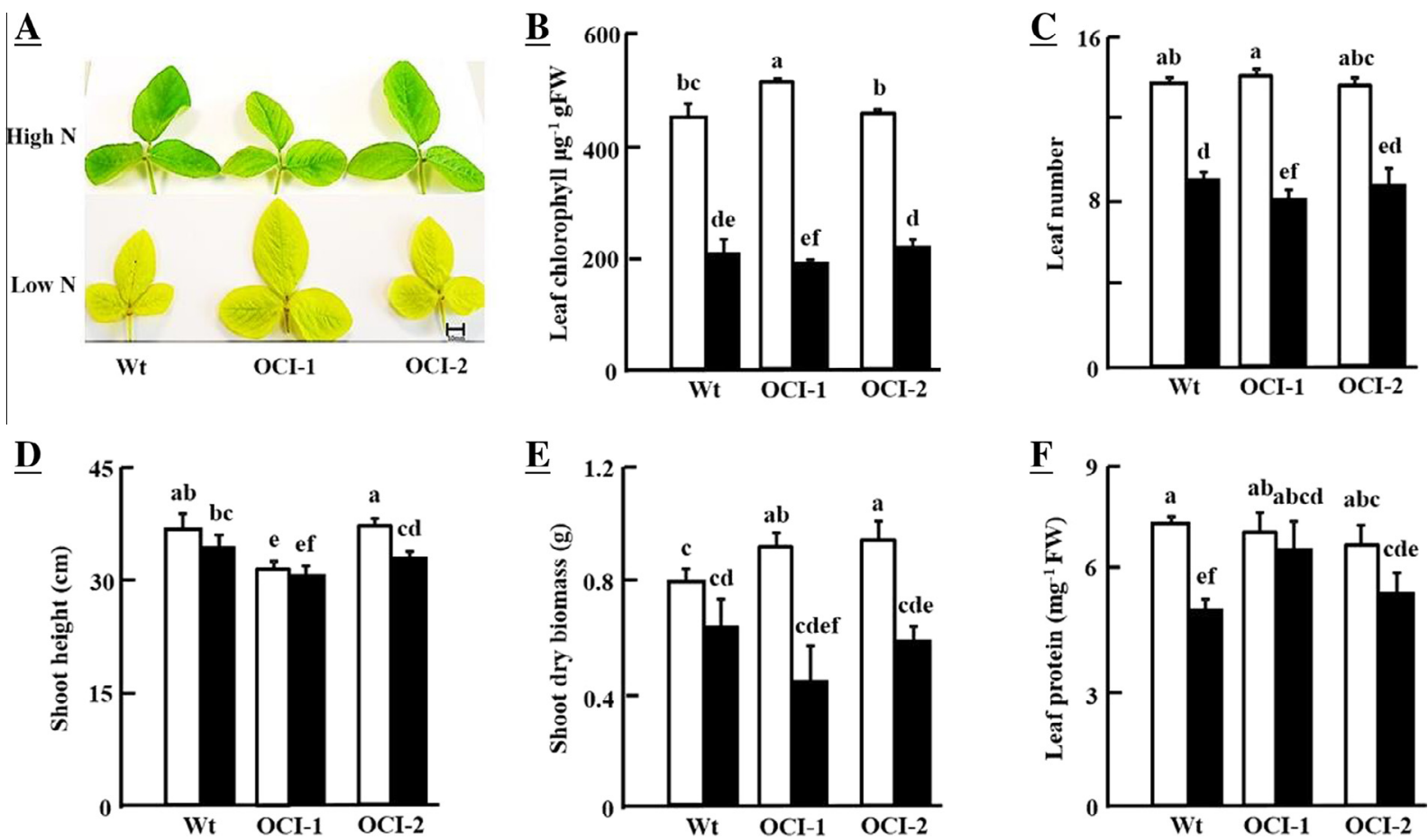

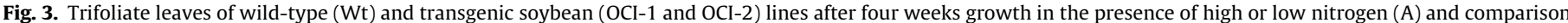

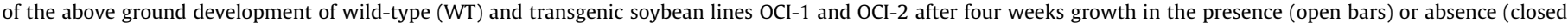

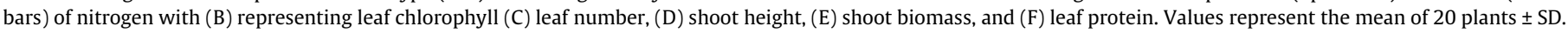
Different letters denote significant differences between the different types of plants at $P \leqslant 0.05$.

$\underline{\mathbf{A}}$

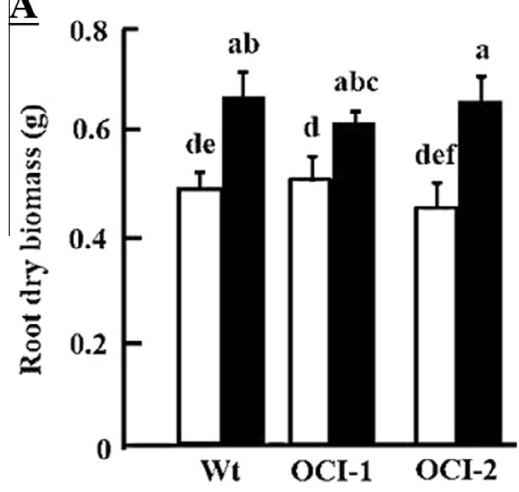

$\underline{B}$

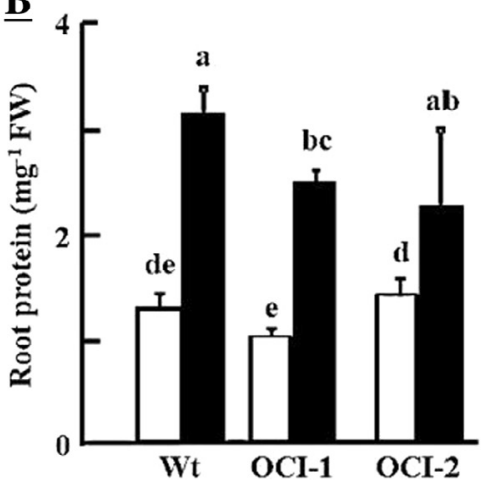

$\underline{\mathrm{C}}$

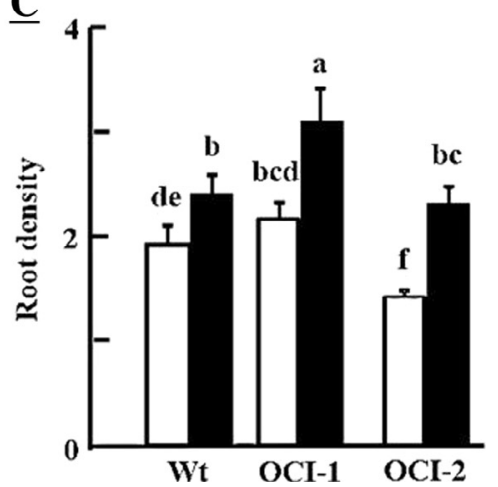

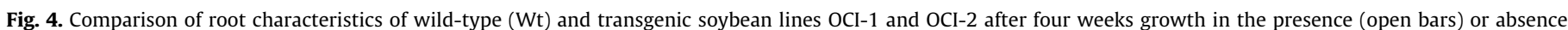

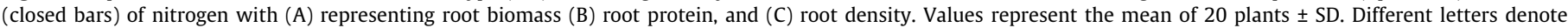
significant differences between the different types of plants at $P \leqslant 0.05$.

was significantly lower $(P \leqslant 0.05)$ under LN compared to HN conditions (Fig. 5F). Transcripts encoding CP4 were reported to be highly expressed in leaves and roots (Table 2). The abundance of leaf (Fig. 4C) and root (Fig. 5G) CP4 transcripts was similar under both nitrogen regimes.

In contrast to papain-like cysteine proteases, abundance of transcripts of cysteine proteases of the VPE type (VPE2 and VPE3) was sensitive to nitrogen availability in both leaves and roots (Fig. 6). Transcripts encoding VPE2 (Fig. 6A), which were not detectable in leaves and poorly expressed in roots, and also VPE3 transcripts (Fig. 6B), which were detectable in leaves and lowly expressed in roots in the RNAseq analysis (Table 2), were significantly $(P \leqslant 0.05)$ higher expressed in leaves and roots of all soybean lines under LN compared to HN conditions, with the exception of VPE3 in the roots of the OCI-2 plants (Fig. 6). VPE2 was much more highly expressed in roots than in leaves.

The responses of transcripts encoding the three different cystatins identified in Table 2 to $\mathrm{LN}$ growth conditions were determined in leaves (Fig. 7A-C) and roots (Fig. 7D-F). Ectopic OCI expression altered the abundance of CYS8 transcripts in leaves. CYS8 transcripts were significantly higher in the OCI- 1 and $\mathrm{OCI}-2$ leaves than those of the wild type under HN conditions (Fig. 7A). The abundance of CYS8 transcripts was significantly decreased in the leaves of all lines under LN conditions compared to plants grown under HN (Fig. 7A). The abundance of CYS8 transcripts in the leaves of OCI- 1 plants grown under LN conditions was significantly higher than in the wild-type leaves (Fig. 7A). 
Table 2

Effects of low nitrogen on the expression of genes encoding vacuolar processing cysteine proteases (VPE), papain-like cysteine proteases and cystatins in soybean.

\begin{tabular}{|c|c|c|c|}
\hline Phytozome ID & Gene expression (leaf) (Severin et al., 2010) & Gene expression (root) (Severin et al., 2010) & Gene expression (leaf and root) (low $N$ ) \\
\hline \multicolumn{4}{|l|}{ VPE } \\
\hline Glyma05g04230 (VPE2) & Non-detectable & Very low & Leaf $\uparrow \operatorname{Root} \uparrow$ \\
\hline Glyma14g10620 (VPE3) & Non-detectable & Low & Leaf $\uparrow$ Root $\uparrow$ \\
\hline \multicolumn{4}{|c|}{ Cysteine protease (papain-like) } \\
\hline Glyma17g05670 (CP2) & Medium & Medium & Leaf $\downarrow$ Root $\downarrow$ \\
\hline Glyma10g35100 (CP3) & Non-detectable & Very low & Leaf $\downarrow$ Root $\downarrow$ \\
\hline Glyma14g40670 (CP4) & Very high & Very high & No change \\
\hline Glyma04g03090 (CP5) & Very low & Very low & Leaf $\downarrow$ Root $\downarrow$ \\
\hline \multicolumn{4}{|l|}{ Cystatin Glyma13g04250 } \\
\hline (CYS8) Glyma14g04260 & High & High & Leaf $\downarrow$ Root $\downarrow$ \\
\hline (CYS9) Glyma18g00690 & Medium & Non-detectable & Leaf $\uparrow$ \\
\hline (CYS10) & Non-detectable & Non-detectable & Leaf $\uparrow$ Root $\uparrow$ \\
\hline
\end{tabular}

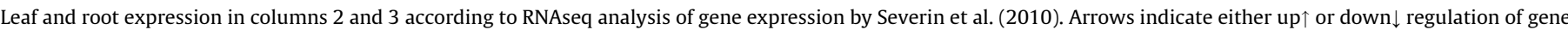
expression when compared to high $N$ treatment.

\section{$\underline{\text { Leaf }}$}
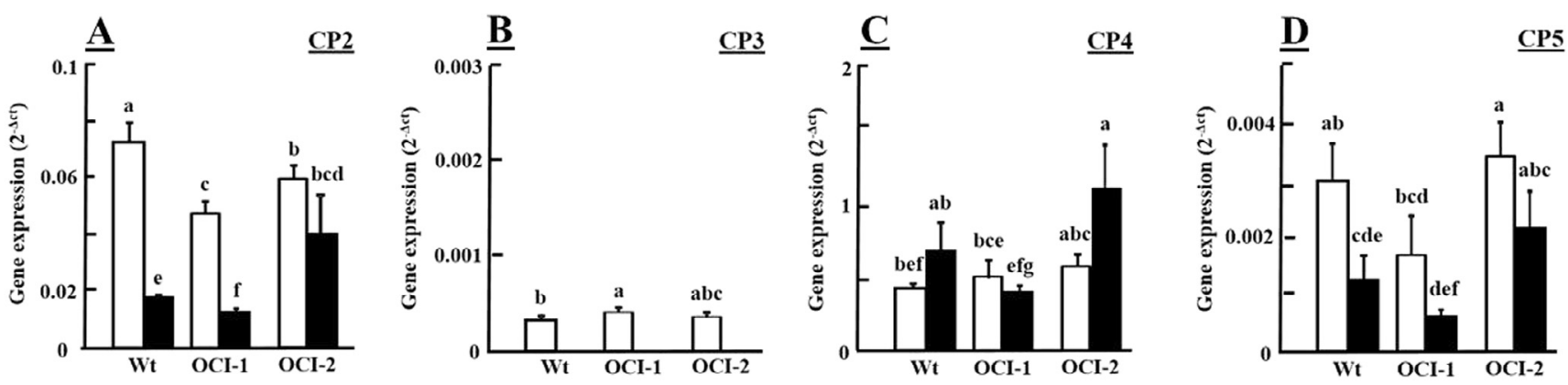

\section{$\underline{\text { Root }}$}
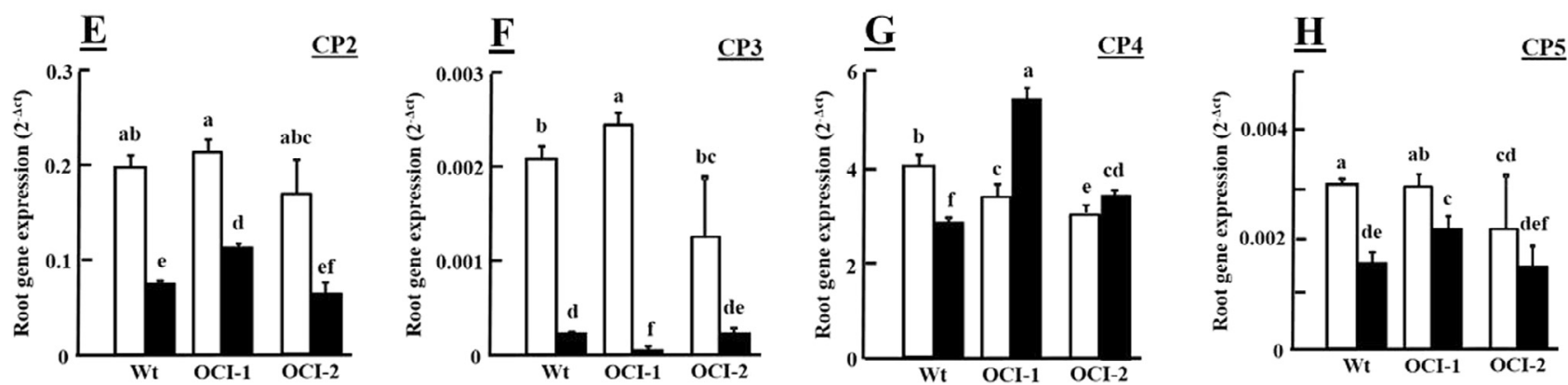

Fig. 5. Comparison of transcription of different papain-like cysteine proteases in leaves and shoots of wild-type (Wt) and transgenic plants of lines OCI-1 and OCI-2 after

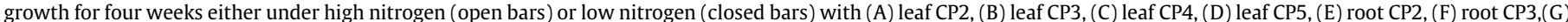
root $\mathrm{CP} 4$ and $(\mathrm{H})$ root $\mathrm{CP} 5$. Values represent the mean of two biological replicates with 3 technical replications per gene \pm SD. Different letters denote significant

differences between the different types of plants at $P \leqslant 0.05$.

In contrast to the LN-dependent decreases in CYS8 transcripts observed in the leaves of all lines (Fig. 7A), the levels of CYS9 (Fig. 7B) and CYS10 (Fig. 7B) transcripts were significantly higher in the leaves of all lines under LN than under HN conditions. Leaf CYS9 transcripts were significantly higher in the OCI-1 and OCI-2 plants than the wild type under HN conditions (Fig. 7B). However, the levels of CYS9 transcripts were significantly lower in the OCI-1 and OCI-2 leaves than the wild type under LN conditions (Fig. 7B). While the abundance of CYS10 transcripts was significantly higher in the OCI- 1 and OCI-2 leaves than in the wild type under HN conditions, the levels of CYS10 transcripts was similar in all lines under the LN regime (Fig. 7C).

The effects of nitrogen nutrition and ectopic OCI expression on the abundance of root CYS8 transcripts (Fig. 7D) showed similar trends to those observed in leaves (Fig. 7A). CYS8 transcripts were significantly higher in the roots of all lines grown under HN compared to LN conditions (Fig. 7D). The abundance of CYS8 transcripts was significantly higher in the $\mathrm{OCI}-1$ and $\mathrm{OCI}-2$ roots than in the wild type under HN conditions. In contrast, CYS8 transcript levels were significantly lower in the $\mathrm{OCI}-1$ roots than in the wild type only under LN conditions (Fig. 7D).

The levels of CYS9 transcripts in the wild-type roots were significantly higher under LN compared to the HN regime (Fig. 7E). CYS9 transcripts were also significantly higher in the roots of the OCI-1 and $\mathrm{OCI}-2$ plants than the wild type under HN conditions (Fig. 7E). However, there was no consistent difference in CYS9 transcripts in the roots of the OCI- 1 and $\mathrm{OCI}-2$ plants relative to the wild type under LN conditions (Fig. 7E). CYS10 transcripts were significantly 
A

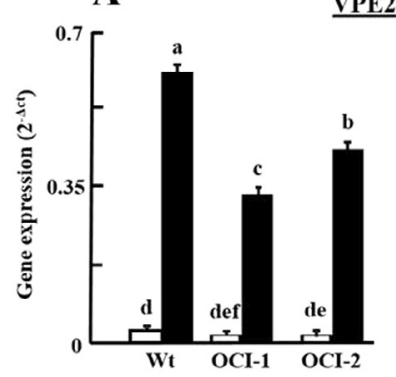

$\underline{\text { Root }}$

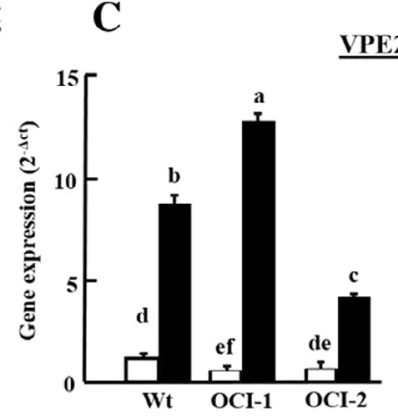

B

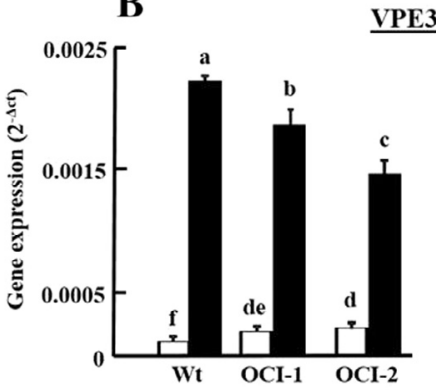

D

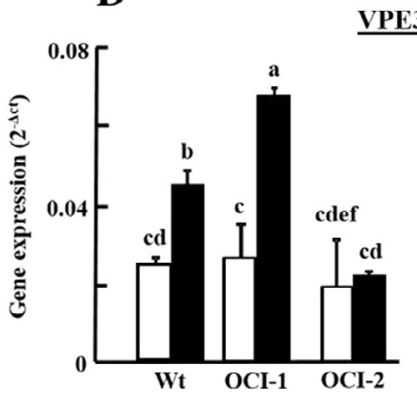

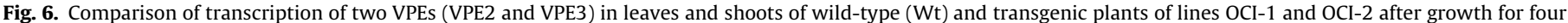

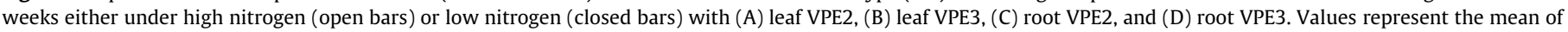
two biological replicates with 3 technical replications per gene \pm SD. Different letters denote significant differences between the different types of plants at $P \leqslant 0.05$.

\section{$\underline{\text { Leaf }}$}
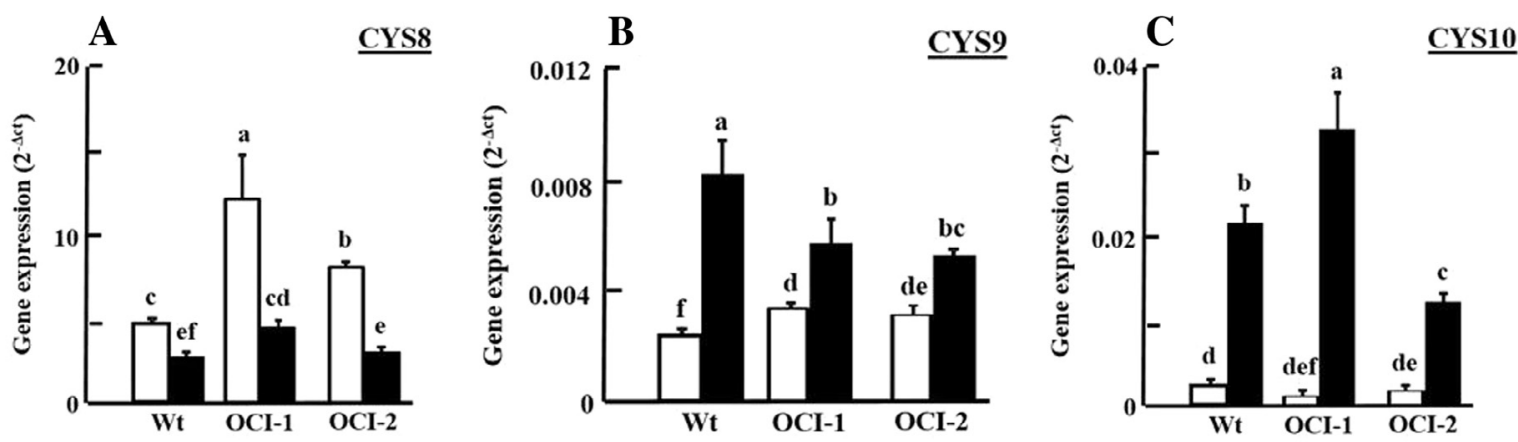

\section{$\underline{\text { Root }}$}
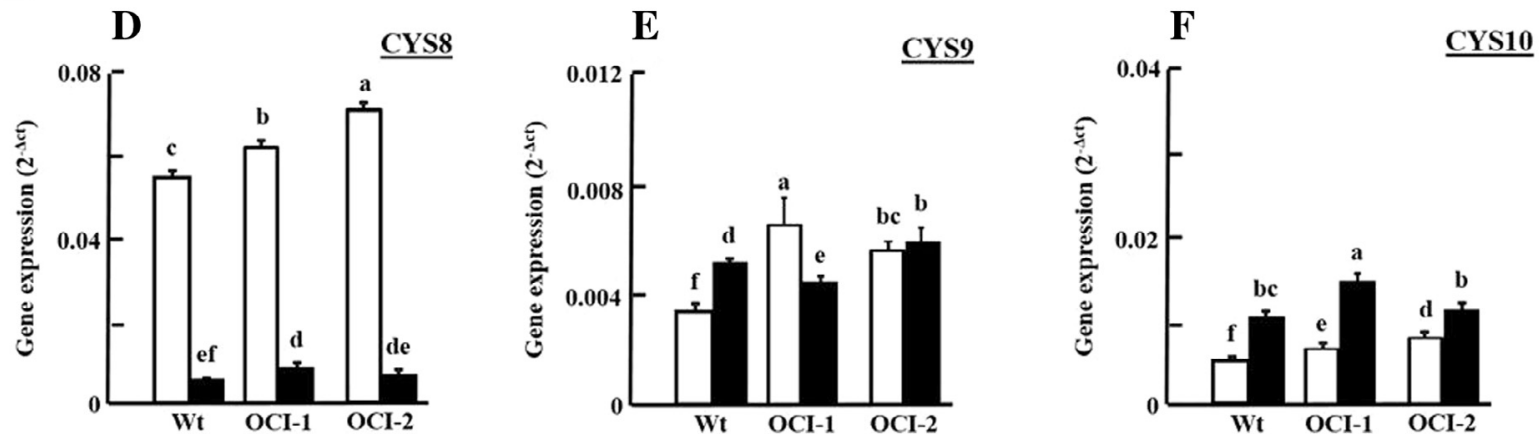

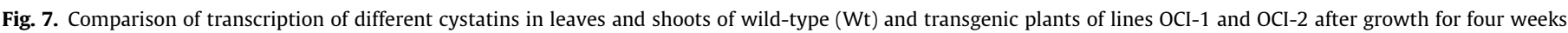

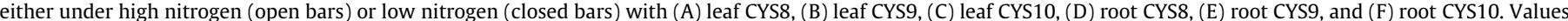

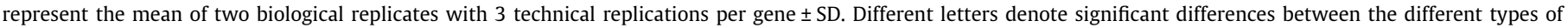
plants at $P \leqslant 0.05$.

higher in the roots of all lines under LN compared to HN conditions (Fig. 7F). The levels of CYS10 transcripts was higher in the OCI-1 and $\mathrm{OCI}-2$ roots than those of the wild type under HN conditions but there was no consistent difference in CYS10 transcripts in the roots of the OCI- 1 and OCI- 2 plants relative to the wild type under LN conditions (Fig. 7F). 


\section{Discussion}

Plants shuttle nitrogen reserves between different plant organs during development, particularly during senescence and seed set. Translocation of accumulated nitrogen reserves from older to newer tissues is achieved by the programmed turnover of cellular components, a process in which protein degradation plays a central role (Agren, 1985; McConnaughay and Coleman, 1999; Lemaire et al., 2008; Kraiser et al., 2011). This mechanism becomes systemic under conditions of nitrogen limitation, due to the need to triage constituents, and completion of the life cycle becomes prioritized over vegetative growth (Carter and Knapp, 2000; Tranbarger et al., 2003; Zhao et al., 2005; Hermans et al., 2006). The composition of cells is changed as organs enter senescence leading to lower protein accumulation, a process that requires the active participation of a large suite of proteases as well as the ubiq-uitin-proteasome system. The data reported here confirm earlier reports that transcripts encoding papain-like cysteine proteases and cystatins are affected (Scheible et al., 2004; Bi et al., 2007). Moreover, the results demonstrate that VPE-like cysteine proteases are likely to be as important in the degradation of leaf and root pro-teins for remobilisation during nitrogen deficiency, as they are in the remobilisation of seed proteins (Shimada et al., 2003).

Leaf chlorophyll and protein were significantly lower in plants grown under nitrogen deficiency compared to HN conditions. LNdependent decreases in leaf and root protein were less marked in the OCI- 1 and $\mathrm{OCI}-2$ lines than the wild type. Unlike plants grown in soil (Quain et al., 2014), similar levels of OCI transcripts were measured in the OCI- 1 and OCI-2 lines grown in vermiculite with nutrient solution. The OCI-1 and OCI-2 lines had significantly higher amounts of shoot biomass than the wild type under HN but not LN conditions. Moreover, the LN-dependent decreases in leaf protein and increases in root protein contents were smaller in the roots of the OCI- 1 and $\mathrm{OCI}-2$ lines than in the wild type. These data show that the LN-dependent responses of the soybean plants are modified in the OCI- 1 and $\mathrm{OCI}-2$ lines, suggesting that cysteine proteases that are inhibited by $\mathrm{OCI}$ are important in the remobilisation of protein from the leaves to the roots in situations of nitrogen deficiency. In addition, the OCI- 1 and OCI- 2 lines had significantly greater numbers of crown nodules than the wild type, demonstrating that cysteine proteases that are sensitive to $\mathrm{OCI}$ mediated inhibition influence the nodulation process. While the present study does not allow the identification of the specific papain-like cysteine proteases that are involved in the remobilisation of leaf protein at LN or in nodulation allowing symbiotic nitrogen fixation, several trends in cysteine protease expression can be identified. For example, the data presented here show that the Asnspecific cysteine protease VPE2, which was used in this study as a representative VPE, plays a role in protein remobilisation in leaves and roots at the later stages of nitrogen limitation.

VPE2 and VPE3 transcripts were increased in leaves of OCIexpressing lines under LN conditions. VPE2 is also involved in protein remobilisation during seed-set (Muntz and Shutov, 2002) but the functions of VPE3, which was also a selected representative VPE used in this study, are poorly characterised according to the SoyBase database (www.soybase.org, accessed at 3.6.2014). The data presented here suggest that VPE3 also plays a role in peptide hydrolysis during LN-induced senescence. Cystatins that inhibit VPEs require a C-terminal extension containing a SNSL amino acid motif, which is not present in OCI (Martinez et al., 2007). Hence OCI, like the cysteine protease inhibitor E64, is a poor inhibitor of VPE activity (Hatsugai et al., 2006). Therefore, the OCI protein expressed in the transgenic soybean plants is unlikely to have a direct inhibitory effect on the endogenous activities of these VPEs. It is possible therefore that other uniden- tified cysteine protease inhibitors influence the expression of the VPEs studied here. Moreover, we were unable to detect significant levels of legumain activity in extracts of soybean plants grown under HN or LN conditions using the fluorescent legumain-like ZAla-Ala-Asn-MCA substrate. This finding suggests that despite the observed differences in the abundance of VPE transcripts under HN or LN conditions, the resultant changes in VPE activity are small and below the levels of detection by the standard enzymatic assay procedures. Transcripts encoding CP3, which is highly expressed in seeds (Severin et al., 2010) were below the level of detection in leaves under LN conditions. This suggests that this cysteine protease does not have an important role in plant adaptation to nitrogen limitation.

Ectopic OCI expression exerted an influence over the abundance of transcripts encoding several cystatins, including CYS8, CYS9 and CYS10, particularly under LN conditions. This finding suggests that papain-like cysteine proteases, which interact with cystatins may be involved in transcription control of cystatin expression. CYS10 transcripts were much more abundant under LN than HN growth conditions. Although this cystatin has a C-terminal extension, it does not contain the characteristic SNSL domain, which is required to control VPE activity. CYS10 is comprised of only 150 amino acids, whereas other possible legumain inhibitors are larger with 240 amino acids. None of the other cystatins selected for our transcription analysis (CYS8 and CYS9) had a C-terminal extension which is required for VPE inhibition. More in-depth studies are required to elucidate whether the expression patterns of cystatins with a SNSL domain are regulated in response to nitrogen availability. Similarly, more studies are required to establish unequivocally that the expression of CYS10 is LN-dependent and whether CYS10 expression is controlled at the later stages of the senescence process. It is possible that OCI expression might have greater benefits at earlier stages of $\mathrm{N}$-limitation than that studied here i.e., before the onset of leaf senescence, when leaves show higher levels of papain-like cysteine proteases. The data presented here show that abundance of transcripts encoding proteases was generally lower under LN conditions. Earlier studies suggest that the proteases that facilitate remobilisation during nutrient deficiency are not the same as those involved during senescence-related nitrogen mobilisation (Kingston-Smith et al., 2005; Yamauchi et al., 2002). Such findings suggest that nitrogen limitation initially induces a reversible senescence process in which proteases facilitate the degradation of non-essential proteins to enhance plant survival (KingstonSmith et al., 2005). Our study has focused on the later stages of LNinduced senescence, which is already characterised by VPE expression and a lower abundance of transcripts encoding papainlike cysteine proteases and cystatins.

The data presented here demonstrate that OCI-dependent inhibition of papain-like cysteine proteases enhanced shoot biomass under HN conditions and reduced the loss of leaf protein in plants growing under LN. While ectopic OCI expression offers only limited protection to soybeans subjected to nitrogen limitation, OCIdependent inhibition of papain-like cysteine proteases clearly exerts an influence over the levels of transcripts encoding VPEs and cystatins, which may be important components of the plant response to nitrogen deficiency. More studies are required to determine how OCI-sensitive cysteine proteases influence the expression and/or turnover of VPEs and cystatin transcripts.

\section{Acknowledgements}

This work was funded by FP7-PIRSES-GA-2008-230830 (LEGIM) and PIIF-GA-2011-299347 (Soylife; K.K.). We thank Leah March for technical assistance. M.Q. thanks the Schlumberger Foundation Faculty for the Future Award for her fellowship. JC thanks BBSRC for a CASE studentship (BB/K501839/1). 


\section{References}

Agren, G.I., 1985. Theory of growth of plants derived from the nitrogen productivity concept. Physiol. Plant. 64, 17-28.

Arai, S., Watanabe, H., Kondo, H., Yemori, E., Abe, K., 1991. Papain-inhibitory activity of oryzacystatin, a rice seed cysteine proteinase inhibitor, depends on the central Gln-Val-Val-Ala-Gly region conserved among cystatin superfamily members. J. Biochem. 109, 294-298.

Beers, E.P., Woffenden, B.J., Zhao, C., 2000. Plant proteolytic enzymes: possible roles during programmed cell death. Plant Mol. Biol. 44, 399-415.

Benchabane, M., Schlüter, U., Vorster, J., Goulet, M.C., Michaud, D., 2010. Plant cystatins. Biochimie 92, 1657-1666.

Bi, Y.M., Wang, R.L., Zhu, T., Rothstein, S.J., 2007. Global transcription profiling reveals differential responses to chronic nitrogen stress and putative nitrogen regulatory components in Arabidopsis. BMC Genomics 8, 281.

Bradford, M.M., 1976. A rapid sensitive method for the quantification of microgram quantities of protein utilizing the principal of protein-dye binding. Anal. Biochem. 72, 248-254.

Carter, G.A., Knapp, A.K., 2000. Leaf optical properties in higher plants, linking spectral characteristics to stress and chlorophyll concentrations. Am. J. Bot. 88, 677-684.

Crafts-Bradner, S.J., Holzer, R., Feller, U., 1998. Influence of nitrogen deficiency on senescence and the amounts of RNA and proteins in wheat leaves. Physiol. Plant. 102, 192-200.

De Bolle, M., Butaye, K., Coucke, W., Goderis, I., Wouters, P., van Boxel, N., Broekaert, W., Cammue, B., 2003. Analysis of the influence of promoter elements and a matrix attachment region on the inter-individual variation of transgene expression in populations of Arabidopsis thaliana. Plant Sci. 165, 169-179.

Esteban-Garcia, B., Garricardenas, J.A., Alonso, D.L., Garcia-Maroto, F., 2010. A distinct superfamily of papain-like cysteine proteinases regulated by senescence and stresses in Glycine max. J. Plant Physiol. 167, 1101-1108.

Forsthoefel, N.R., Cushman, M.A.F., Ostrem, J.A., Cushman, J.C., 1998. Induction of a cysteine protease cDNA from Mesembryanthemum crystallinum leaves by environmental stress and plant growth regulators. Plant Sci. 136, 195-206.

Gordon, A.J., Kessler, W., Minchin, F.R., 1990. Defoliation-induced stress in nodules of white clover. I. Changes in physiological parameters and protein synthesis. J. Exp. Bot. 41, 1245-1253.

Graham, P.H., Vance, C.P., 2000. Nitrogen fixation in perspective: an overview of research and extension needs. Field Crops Res. 65, 93-106.

Graham, P.H., Vance, C.P., 2003. Legumes: importance and constraints to greater use. Plant Physiol. 131, 872-877.

Grudkowska, M., Zagdanska, B., 2004. Multifunctional role of plant cysteine proteinases. Acta Biochim. Pol. 51, 609-624.

Hara-Nishimura, I., Hatsugai, N., Nakaune, S., Kuroyanagi, M., Nishimura, M., 2005. Vacuolar processing enzyme: an executor of plant cell death. Curr. Opin. Plant Biol. 8, 404-408.

Hatsugai, N., Kuroyanagi, M., Nishimura, M., Hara-Nishimura, I., 2006. A cellular suicide strategy of plants: vacuole-mediated cell death. Apoptosis 11, 905-911.

Hermans, C., Hammond, J.P., White, P.J., Verbruggen, N., 2006. How do plants respond to nutrient storage by biomass allocation? Trends Plant Sci. 11, 610-617.

Horstensteiner, S., Feller, U., 2002. Nitrogen metabolism and remobilization during senescence. J. Exp. Bot. 53, 927-937.

Jenko, S, Dolenc, I., Guncar, G., Dobersek, A, Podobnik, M., Turk, D, 2003. The refined 2.4 A X-ray crystal structure of recombinant human stefin B in complex with the cysteine proteinase papain: a novel type of proteinase inhibitor interaction. J. Mol. Biol. 326, 875-885.

Jeuffroy, M.H., Ney, B., 1997. Crop physiology and productivity. Field Crops Res. 53, $3-16$.

Kant, S., Bi, Y.M., Rothstein, S.J., 2010. Understanding plant response to nitrogen limitation for the improvement of crop nitrogen use efficiency. J. Exp. Bot. 62 1499-1509.

Karina, B.B., Benavides, M.P., Gallego, S.M., Tomaro, M.L., 2003. Effect of cadmium stress on nitrogen metabolism in nodules and roots of soybean plants. Funct. Plant Biol. 30, 57-64.

Kato, H., Sutoh, K., Minamikawa, T., 2003. Identification, cDNA cloning and possible roles of seed-specific rice asparaginyl endopeptidases, REP-2. Planta 217, 676-685.

Kingston-Smith, A.H., Bollard, A.L., Minchin, F.R., 2005. Stress-induced changes in protease composition are determined by nitrogen supply in non-nodulating white clover. J. Exp. Bot. 56, 745-753.

Kraiser, T., Gras, D.E., Gutierrez, A.G., Gonzalez, B., Gutierrez, R.A., 2011. A holistic view of nitrogen acquisition in plants. J. Exp. Bot. 62, 1455-1466.

Lemaire, G., Jeuffroy, M.H., Gastal, F., 2008. Diagnosis tool for plant and crop N status in vegetative stage: theory and practices for crop $\mathrm{N}$ management. Eur. J. Agron. 28, 614-624.

Lichtenthaler, H.K., 1987. Chlorophylls and carotenoids - pigments of photosynthetic biomembranes. Methods Enzymol. 148, 350-382.

Martinez, M., Diaz, I., 2008. The origin and evolution of plant cystatins and their target cysteine proteinases indicate a complex functional relationship. BMC Evol. Biol. 8, 198
Martinez, M., Diaz-Mendoza, M., Carrillo, L., Diaz, I., 2007. Carboxy terminal extended phytocystatins are bifunctional inhibitors of papain and legumain cysteine proteinases. FEBS Lett. 581, 2914-2918.

Martinez, M., Cambra, I., Gonzalez-Melendi, P., Santamaria, M.E., Diaz, I., 2012. C1A cysteine-proteases and their inhibitors in plants. Physiol. Plant. 145, 85-94.

McConnaughay, K.D.M., Coleman, J.S., 1999. Biomass allocation in plants: ontogeny or optimality? A test along three resource gradients. Ecology 80, 2581-2593.

Mengel, D., Ruiz-Diaz, D.A., 2012. Applying nitrogen to N-stressed soybeans. Updated: 06/22/2012. <http://extension.agron.iastate.edu/soybean/production soilfert.html>.

Moulin, L., Munive, A., Dreyfus, B., Boivin-Masson, C., 2001. Nodulation of legumes by members of the $\beta$-subclass of proteobacteria. Nature 411, 948-950.

Muntz, K., Shutov, A.D., 2002. Legumains and their function in plants. Trends Plant Sci. 7, 340-344

Oldroyd, G.E.D., Murray, J.D., Poole, P.S., Downie, J.A., 2011. The rules of engagement in the legume-rhizobial symbiosis. Annu. Rev. Genet. 45, 119-144.

Park, J.R., McFarlane, I., Phipps, R.H., Ceddia, G., 2011. The role of transgenic crops in sustainable development. Plant Biotech. J. 9, 2-21.

Pellny, T.K., Locato, V., Vivancos, P.D., Markovic, J., De Gara, L., Pallardó, F.V., Foyer, C.H., 2009. Pyridine nucleotide cycling and control of intracellular redox state in relation to poly (ADP-ribose) polymerase activity and nuclear localization of glutathione during exponential growth of Arabidopsis cells in culture. Mol. Plant 2, 442-456.

Prins, A., Van Heerden, P.D.R., Olmos, E., Kunert, K.J., Foyer, C.H., 2008. Cysteine proteinases regulate chloroplast protein content and composition in tobacco leaves: a model for dynamic interactions with ribulose-1, 5-bisphosphate carboxylase/oxygenase (Rubisco) vesicular bodies. J. Exp. Bot. 59, 19351950.

Quain, M.D., Makgopa, M.E., Marquez-Garcia, B., Comadira, G., Fernandez-Garcia, N., Olmos, E., Schnaubelt, D., Kunert, K.J., Foyer, C.H., 2014. Ectopic phytocystatin expression leads to enhanced drought stress tolerance in soybean (Glycine max) and Arabidopsis thaliana through effects on strigolactone pathways and can also result in improved seed traits. Plant Biotech. J. http://dx.doi.org/10.1111/ pbi.12193.

Roberts, I.N., Caputo, C., Criado, M.V., Funk, C., 2012. Senescence-associated proteases in plants. Physiol. Plant. 145, 130-139.

Salvesen, G., Nagase, H., 1989. Inhibition of proteolytic enzymes. IRL Press, New York.

Scheible, W.R., Morcuende, R., Czechowski, T., Fritz, C., Osuna, D., Palacios-Rojas, N., Schindelasch, D., et al., 2004. Genome-wide reprogramming of primary and secondary metabolism, protein synthesis, cellular growth processes, and the regulatory infrastructure of Arabidopsis in response to nitrogen. Plant Physiol. 136, 2483-2499.

Schmutz, J., Cannon, S.B., Schlueter, J., Ma, J., Mitros, T., Nelson, W., Hyten, D.L., et al., 2010. Genome sequence of the palaeopolyploid soybean. Nature 463, 178-183.

Severin, A.J., Woody, J.L., Bolon, Y.T., Joseph, B., Diers, B.W., Farmer, A.D., Muehlbauer, G.J., et al., 2010. RNA-Seq atlas of Glycine max: a guide to the soybean transcriptome. BMC Plant Biol. 10, 160.

Shimada, T., Yamada, K., Kataoka, M., Shimada, T., Nakaune, S., Koumoto, Y., Kuroyanagi, M., et al., 2003. Vacuolar processing enzymes are essential for proper processing of seed storage proteins in Arabidopsis thaliana. J. Biol. Chem. 278, 32292-32299.

Solomon, M., Belenghi, B., Delledonne, M., Menachem, E., Levine, A., 1999. The involvement of cysteine proteases and protease inhibitor genes in the regulation of programmed cell death in plants. Plant Physiol. 11, 431-443. Tajima, T., Yamaguchi, A., Matsushima, S., Satoh, M., Hayasaka, S., Yoshimatsu, K., Shioi, Y., 2011. Biochemical and molecular characterization of senescence-related cysteine protease-cystatin complex from spinach leaf. Physiol. Plant. 141, 97116.

Tilman, D., Balzer, C., Hill, J., Befort, B.F., 2011. Global food demand and the 20264.

Tranbarger, T.J., Al-Ghazi, Y., Muller, B., Teyssendier de la Serve, B., Doumas, P., Touraine, B., 2003. Transcription factor genes with expression correlated to nitrate-related root plasticity of Arabidopsis thaliana. Plant Cell Environ. 26, 459-469.

Van der Vyver, C., Schneidereit, J., Driscoll, S., Turner, J., Kunert, K., Foyer, C.H., 2003 Oryzacystatin I expression in transformed tobacco produces a conditional growth phenotype and enhances chilling tolerance. Plant Biotech. J. 1, 101112

Van Heerden, P.D.R., Kruger, G.H.J., Loveland, J.E., Parry, M.A.J., Foyer, C.H., 2003. Dark chilling imposes metabolic restrictions on photosynthesis in soybean. Plant Cell Environ. 26, 323-337.

Yamauchi, Y., Suimoto, T., Sueyoshi, K., Oji, Y., Tanaka, K., 2002. Appearance of endopeptidases during the senescence of cucumber leaves. Plant Sci. 162, 615619.

Zhao, D., Reddy, K.R., Kakani, V.G., Reddy, V.R., 2005. Nitrogen deficiency effects on plant growth, leaf photosynthesis, and hyperspectral reflectance properties of sorghum. Eur. J. Agron. 22, 391-403. 